\title{
Synthesis of Arbitrary Group Delay Responses with All-Pass Optical Cavities Structures
}

\author{
Miguel A. Preciado, ${ }^{1, *}$ Xuewen Shu, ${ }^{1}$ Kate Sudgen, ${ }^{1}$ Miguel A. Muriel $^{2}$ \\ ${ }^{1}$ Photonics Research Group, Aston University, Birmingham, B4 7ET, UK. \\ ${ }^{2}$ ETSI Telecomunicacion, Universidad Politecnica de Madrid (UPM), 28040 Madrid, Spain. \\ 'Corresponding author:m.preciado@aston.ac.uk
}

\begin{abstract}
We propose a systematic method for the synthesis of arbitrary group delay responses by using allpass structures of coupled optical cavities. Optimum structure parameters design, in terms of filter order and accuracy, are obtained.

OCIS codes: $140.4780,060.4510,230.1150,350.4600$
\end{abstract}

\section{Introduction}

It is well-known that all-pass optical cavities structures, also known as Gires-Tournois etalon-based structures, are highly suitable in WDM multichannel systems, since their response is spectrally periodic, and can be implemented in a number of technological options. Concretely, Fiber Bragg grating (FBG) implementation [1] offers an in-fiber and inexpensive solution. Phase-only filtering designs of different complexity has been reported for applications of dispersion compensation and slow light [2,3], and systematic techniques for design of optical filters for amplitude filter design, based on that of electronic filters design, are very well-known [3,4]. However, to the best of our knowledge, no similar systematic methods for the design of the parameters of these all-pass optical structures has been reported in detail in order to synthesize an arbitrary group delay response, since only multidimensional nonsystematic optimization algorithms have been reported to be used for the phase-only filter design [3].

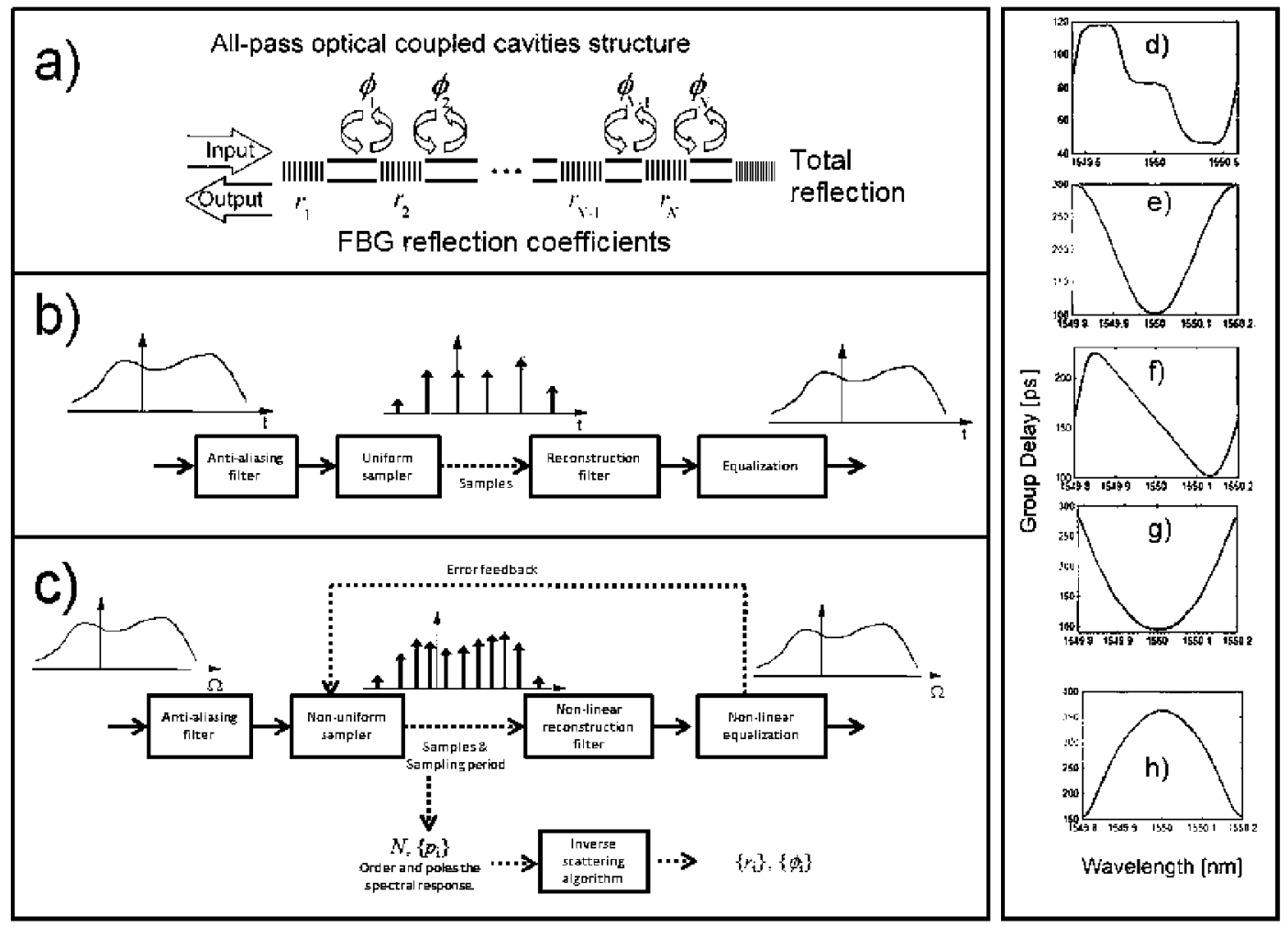

Fig. 1 FBG optical cavities structure (a). Classic signal sampling and reconstruction process (b). Proposed method schematic based on classic signal sampling and reconstruction process (c). Examples of design for slow light delay steps (d), sinusoidal group delay profile (e), linear (f) and quadratic ( $g$ and $h$ ) group delay responses.

We present a novel systematic method to design an all-pass optical structure with coupled cavities as shown in Fig. 1a, for spectrally periodic phase-only filtering with arbitrary group-delay responses. There are some important advantages of using a systematic method. First, since the search space increase with the order of the filter, an nonsystematic optimization algorithm is not practical for high order filters. Second, a systematic method directly 
provides an optimum solution in terms of filter order or accuracy, while an non-systematic optimization may easily lead to a local optimum solution, especially for high orders filters.

\section{Proposed method.}

The group delay response of an all-pass optical cavity structure of order $\mathrm{N}$, composed by $\mathrm{N}$ optical cavities, can be expressed as the superposition of $\mathrm{N}$ first-order group delay functions, which spectral response is composed by a pair pole/zero of values $p_{1}$ and $1 / p_{1}^{*}$ for the $\mathrm{i}$-th component. Each first-order group delay function can be decomposed in a constant value, which can be neglected since it only contributes as a pure delay, and an resonant peak centred at the corresponding resonant frequency with a spectral width FWHMi=4arctan $\left(\sigma_{1}^{-1}\right)$, where $\sigma=\left(1+\left|p_{i}\right|\right) /\left(1-\left|p_{1}\right|\right)$.

The novel method proposed here defines a systematic process to combine these resonant peaks, where the overlapped peaks produce a desired group delay response in an optimum way, in terms of filter order and accuracy. The method is based in a classic sampling and reconstruction of signals, which is adapted to this particular problem, since the function to be sampled and reconstructed is in spectral domain, and it is spectrally periodic (see Fig. $1 \mathrm{~b}$ and 1c). Also, the sampling period is not uniform, and it is modified in order to keep uniform group delay ripples amplitude in a similar way as Chebyshev or elliptic amplitude filter design keep uniform amplitude response ripples in the synthetized spectral amplitude response $[3,4]$. The non-uniform sampler calculates the i-th variable sampling period, $D_{1}$, in order to get uniform amplitude group delay ripples in the reconstructed function, since narrower resonant peaks needs to be placed closer than wider resonant peaks. The reconstruction from the samples is obtained by overlapping the group delay resonant peaks, where every sample corresponds to a different resonant peak. In a similar way as in classic sampling and reconstruction process, an equalization needs to be performed in order to compensate the distortion of the whole process, by emphasizing the high frequency components. In the method proposed, the equalization is performed by a feedback of the error in the reconstructed group delay function.

As a result, from the $\mathrm{N}$ group delay samples and the $N$ sampling periods we can directly obtain the $\mathrm{N}$ poles and $N$ zeros of the spectral response, and then the optical structure parameters $\left\{r_{i}\right\}$ (reflection coefficients) and $\left\{\phi_{1}\right\}$ from an inverse scattering algorithm. The order $N$ of the resulting structure depends on the specified maximum group delay ripples (accuracy), and the desired group delay profile itself.

\section{Examples and conclusions.}

Several examples of the synthesis of group delay profiles have been designed and numerically simulated, where we assume a central wavelength of $1550 \mathrm{~nm}$, and WDM channels of $50 \mathrm{GHz}$. In the first example, the FSR of the filter is $150 \mathrm{GHz}$, which covers three WDM channels, and it is designed to apply a different delay to every channel (slow light). In the second example, a sinusoidal group delay profile with $\mathrm{FSR}=50 \mathrm{GHz}$ is designed to show the capability of obtaining arbitrary profile shapes. In the third example a linear group delay filter with FSR=50 GHz, corresponding to a dispersion value of $500 \mathrm{ps} / \mathrm{nm}$, is designed. In the fourth and fifth example, a quadratic group delay filter with $\mathrm{FSR}=50 \mathrm{GHz}$, corresponding to a dispersion value linear variation of $\pm 2500 \mathrm{ps} / \mathrm{nm}$ (opposite sign in each example), are designed.

The resulting filters have respectively orders $N=12,10,8,8$ and 14 , which also indicates the number of cavities required. The group delay obtained by numerical simulation is respectively showed in Fig. 1.d to 1.h. We want to remark the accuracy of the solutions in most of the total FSR range. Also note the high value of dispersion designed in the third to fifth examples, which cannot be achieved with lower order structures for an FSR of $50 \mathrm{GHz}$. Since the search space dimension is double of the number of cavities (two parameters per cavity), the search space dimensions in these examples are around 20, which is a huge dimensions number for non-systematic optimization methods.

\section{References}

1. X. Shu, K. Sugden, and I. Bennion, "Dual-direction Gires-Tournois etalon based on a single complex fiber Bragg grating," Opt. Lett. 31, 2263-2265 (2006)

2. G. Lenz, B. J. Eggleton, C. K. Madsen, and R. E. Slusher "Optical delay lines based on optical filters," IEEE J.Quantum Electron. 37 , 525$532(2001)$

3. C. K. Madsen and J. H. Zhao, Optical Filter Design and Analysis: A Signal Processing Approach (Wiley, 1999)

4. K. L. Su, "The Approximation,", in Analog Filters (Springer, 2002)

5. J. Proakis and D. G. Manolakis, Digital Signal Processing, (Prentice Hall, 2009)

6. R. Feced, M. N. Zervas, and M. A. Muriel, "An efficient inverse scattering algorithm for the design of nonuniform fiber Bragg gratings," IEEE J. Quantum Electron. 35, 1105-1115 (1999)

This work was supported by the European Comission FP7-PEOPLE-2010-IEF Marie-Curie Action under project "IFOCS", and the Ministerio de Ciencia e Innovacion of Spain under proyect "Plan Nacional de I+D+I TEC2010-2103-C04-02". 